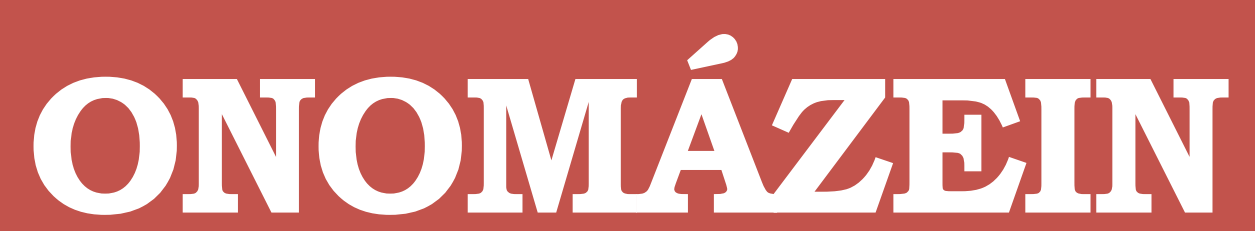

Revista de lingüística, filología y traducción
PONTIFICIA UNIVERSIDAD CATÓLICA DE CHILE FACULTAD DE LETRAS

\title{
Artículos de periodismo automatizado: ¿discurso o no?*
}

Automated journalism articles: discourse or not?

\section{Eirini Chatzikoumi}

Pontificia Universidad Católica de Valparaíso Chile

\section{(C) $($ (i) $\ominus$}

Eirini Chatzikoumi: Departamento de Traducción, Instituto de Literatura y Ciencias del Lenguaje, Pontificia Universidad Católica de Valparaíso, Chile. | E-mail: chatzikoumi@gmail.com 


\section{Resumen}

Dada la centralidad del papel del sujeto en el análisis del discurso, por un lado, y el auge del periodismo automatizado (PA), por otro, se plantea la pregunta de si los productos del PA constituyen discurso, ya que en ellos la existencia de un sujeto claramente definido e incuestionable no queda en evidencia a primera vista y dicha existencia es considerada por el análisis del discurso como requisito indispensable para el discurso. Para responder a esta pregunta, se revisa la bibliografía relacionada con la expresión de la subjetividad en el discurso, la cual se contrasta con una breve revisión bibliográfica acerca de la naturaleza y las características de los productos del PA. La primera revisión permite la descomposición del concepto de subjetividad en una serie de elementos, y el contraste con la segunda, la determinación de la presencia o ausencia de los componentes de la subjetividad en los productos del PA. De esta manera, se responde la pregunta planteada y se proponen futuros estudios de investigación.

Palabras clave: análisis del discurso; sujeto; subjetividad; valoración; generación de lenguaje natural.

\section{Abstract}

Given the central role of the subject in discourse analysis on the one hand, and the rise of automated journalism on the other, the question arises, "can the outputs of automated journalism be considered discourse?", for the existence of a clearly defined and unquestionable subject in them is not evident at first sight, and this existence is for discourse analysis an indispensable requisite of discourse. In order to answer this question, the literature related to the expression of subjectivity in discourse is reviewed and then contrasted with a brief review of the literature related to the nature and features of automated journalism outputs. The first review allows the decomposition of the concept of subjectivity into a series of elements, while the contrast with the second one allows the determination of the presence or absence of these subjectivity components in the automated journalism outputs. Therefore, the question is answered and future research is suggested.

Keywords: discourse analysis; subject; subjectivity; appraisal; natural language generation.

* La autora del artículo fue beneficiaria de Becas Postgrado PUCV 2018 durante su elaboración. 


\section{Introducción}

Dentro del campo de la generación de lenguaje natural, la generación automática de artículos periodísticos es una de las áreas que conocen auge y plantean temas relacionados con la autoría de estos. Este problema de autoría constituye su punto de convergencia con el análisis del discurso, dada la primordialidad que otorga al sujeto en el discurso al punto de considerarlo como requisito indispensable para que una instancia del lenguaje sea caracterizada como discurso. Aparte de la discusión que se está llevando a cabo desde el ámbito del periodismo y en complementación de ella, se estima necesario proporcionar una respuesta desde la lingüística, y concretamente desde el análisis del discurso, a la pregunta: ¿los artículos generados por máquina constituyen discurso? Esta respuesta significa un doble aporte. En primer lugar, permite contribuir al posicionamiento del análisis del discurso acerca de un tipo de productos lingüísticos que podrían considerarse objeto de su estudio o no. Esto, a su vez, puede contribuir al debate periodístico desde la lingüística, de manera que haya una postura más acerca de la autoría de los artículos generados por máquina, tema de suma importancia por su relación con otros asuntos de orden ético y profesional, como el de derechos de autor y la responsabilidad legal. Para ello, se proporciona primero el marco teórico, en el que se detalla la centralidad del sujeto en el análisis del discurso y el marco en el que se incribe el periodismo automatizado (PA) así como su situación actual. Posteriormente, en la sección 3 se procede a la revisión bibliográfica del concepto de la expresión de la subjetividad en el discurso desde distintos enfoques y teorías, y en la sección 4 se desarrolla el problema de la autoría de los productos del PA tras una breve caracterización de ellos en cuanto a calidad lingüística y percepción de parte de humanos. Después de la revisión bibliográfica comparativa, se procede a la discusión del tema y se responde la pregunta planteada. El artículo cierra con unas breves conclusiones.

\section{El sujeto en el análisis del discurso y el estado actual del PA}

Si bien se podría decir del concepto de discurso que es multiforme, en el marco del análisis del discurso se ha visto delimitado por una serie de factores concebidos de una manera determinada, lo que permite su análisis bajo un punto de vista concreto. A nivel de la teoría del discurso, los tres conceptos fundamentales que suelen considerarse como ejes son el poder, el conocimiento y la subjetividad; son los tres factores que, vistos desde la perspectiva del análisis del discurso, forman el triángulo de la teoría del discurso (Angermuller y otros, 2014). Dicha perspectiva funciona con la constante de la construcción de las relaciones, de las identidades y de los roles sociales por medio de las prácticas discursivas y en ellas. En ese construir de relaciones, identidades y roles sociales, se construye también la legitimidad del sujeto a través de la delimitación de quién puede decir qué y desde qué posición. Esto destaca la centralidad de la subjetividad y del sujeto mismo en el discurso.

Por lo tanto, en el marco del análisis del discurso, la subjetividad cobra una naturaleza de omnipresencia. La inseparabilidad de las nociones de sujeto, lenguaje y sociedad se obser- 
va en diversas escuelas, teorías y enfoques. Por un lado, los representantes de la escuela francesa del análisis del discurso destacan el fuerte vínculo entre sujeto y lenguaje así como entre lenguaje y aspecto social, afirmando que la identidad se construye en el discurso, que los objetivos, roles e identidades de los sujetos determinan su comportamiento lingüístico (Charaudeau, 2005), y que el hombre se constituye como sujeto dentro del lenguaje y por medio de él (Benveniste, 1976). Por otro lado, la noción del sujeto como miembro de una sociedad, comunidad o subcultura y al mismo tiempo con un papel fundamental en el discurso es recurrente también en Thompson y Hunston (2001) y su elaboración del concepto de evaluación.

Por otro lado, la generación de lenguaje natural es una subárea del procesamiento del lenguaje natural, que, a su vez, se inscribe en el marco tanto de la inteligencia artificial como de la lingüística computacional. Su objetivo es producir sistemas computacionales que puedan generar textos comprensibles en lenguas humanas (Reiter y Dale, 2000). Caswell y Dörr (2018) la definen como "la creación automática de textos a partir de datos estructurados digitales", estructurados en el sentido de un formato manejable por el computador. Estos sistemas son creados o bien con el objetivo de poder funcionar de manera autónoma sin necesidad de interferencia humana o con el objetivo de servir de asistentes generando un borrador que será modificado por un autor humano (Reiter y Dale, 2000). En el primer caso, Reiter y Dale (2000) se refieren al sistema como autor, y en el segundo, al sistema como asistente de autoría.

La generación de lenguaje natural en el periodismo se realiza en la mayoría de los casos con la técnica de plantillas en las que se introducen palabras a partir de datos estructurados (Fanta, 2017), aunque también existen aproximaciones más sofisticadas, en las que además se analizan los datos (Graefe, 2016). Para estos proyectos es necesaria la colaboración de ingenieros, periodistas y lingüistas computacionales, aunque la tecnología usada es producida por empresas que no se especializan en el periodismo sino en producir sistemas que se puedan aplicar en datos de cualquier ámbito (Graefe, 2016).

La generación de lenguaje natural, junto con otros métodos de automatización, como la detección de noticias y la producción automática de videos, ha venido a transformar el periodismo y dar lugar al fenómeno conocido hoy en día como periodismo automatizado, el cual pertenece al campo del periodismo computacional (Graefe, 2016). Se ha usado una variedad de términos para referirse a este fenómeno: periodismo cuantitativo (Caswell y Dörr, 2018), periodismo automatizado o algorítmico y robot journalism o robojournalism (Montal, 2015; Graefe, 2016; Caswell y Dörr, 2018), término que en español se conoce como periodismo-robot o periodismo robótico; por extensión, también se habla de reportero robot y de robot-periodista. Se trata del proceso de generación automática de noticias con el uso de programas computacionales sin la intervención humana (Graefe, 2016), es decir, lo que Reiter y Dale (2000: 6) denominan "computador como autor". En este artículo se usará el término periodismo automatizado (PA) como el más recurrente en la bibliografía revisada. 
Hace aproximadamente cincuenta años que surgió el PA y el campo originario fue el de los pronósticos del tiempo (Reiter y Dale, 2000; Graefe, 2016). En el ámbito hay consenso acerca de su futuro prometedor a pesar de su campo de aplicación todavía limitado (Montal, 2015; Graefe, 2016; Fanta, 2017). En 2016, Graefe estaba confirmando que se estaban desarrollando sistemas de generación automática de noticias en todo el mundo, mencionando medios como Forbes, The New York Times y Los Angeles Times. En el año 2017, según Fanta (2017), en Europa y EEUU, nueve agencias de noticias, incluidos Reuters y Associated Press, ya estaban usando PA, y dos más estaban en proceso de preparación o desarrollo de sistemas para implementarlo. En ese momento, la Associated Press estaba generando reportajes sobre informes económicos para 3.700 empresas, y Reuters y AFP, miles de reportajes automatizados al año (Fanta, 2017).

Dado que para la generación automática de noticias es necesaria la disponibilidad de datos estructurados, los campos en los que más se ha desarrollado son los que más datos fiables de este tipo poseen: economía, deportes y tiempo (Montal, 2015; Graefe, 2016; Fanta, 2017; Caswell y Dörr, 2018), aunque también la ha habido en resultados de elecciones (Leppännen y otros, 2017), datos numéricos oficiales sobre la educación, el desempleo y la inmobiliaria - gracias a fuentes oficiales que comparten sus datos en formatos adecuados (Fanta, 2017)—, con el campo más innovador, el de la narración de eventos basada en datos policiales (Fanta, 2017); Graefe (2016) hace referencia concretamente a noticias sobre homicidios y terremotos, y Caswell y Dörr (2018) proponen dos métodos para generar narrativas periodísticas.

Las causas de las limitaciones del periodismo automatizado para la mayoría de los sistemas existentes se podrían resumir en las siguientes: falta de capacidad de comparación entre datos nuevos y anteriores, alto costo de desarrollo y mantenimiento de sistemas y de datos, dependencia de datos externos, limitaciones técnicas de las agencias, vacilación de las agencias ante la innovación tecnológica (Fanta, 2017), falta de datos adecuados que permitirían la expansión en otro tipo de noticias (Caswell y Dörr, 2018), falta de cobertura en campos para los que no se dispone de datos estructurados o datos de buena calidad, incapacidad de plantear preguntas, explicar fenómenos nuevos y establecer relaciones de causalidad (Graefe, 2016). Por tanto, a pesar de ser un campo en auge, todavía se ve limitado en términos de complejidad y rango de cobertura (Fanta, 2017) así como a nivel de tareas periodísticas más complejas, como orientación y formación de opinión pública (Graefe, 2016), además de calidad lingüística, la cual será desarrollada en la sección 4.1.

\section{Expresión de subjetividad en el discurso}

En esta sección se revisa la expresión de la subjetividad en el discurso desde distintas teorías, escuelas y enfoques. Por “subjetividad” se entiende 'opinión', 'sentimientos', 'actitud', 'postura' y 'punto de vista' del sujeto, y en la bibliografía se puede encontrar bajo distintas denominaciones: evaluación, valoración, afecto, entre otras. La selección del término dependerá del autor, y las características que se le atribuyen a cada uno de estos conceptos presentan menor o mayor solapamiento según el autor y la teoría. 
Primero, se presenta la teoría de la valoración de John Dewey (2008 [1977]); a continuación, se revisa el concepto de subjetividad en la escuela francesa del análisis del discurso con especial énfasis en la noción de ethos de Maingueneau (2002); la tercera parte de la revisión está dedicada a la teoría de la valoración en el marco de la lingüística sistémico funcional (LSF); la cuarta, al concepto de evaluación según Thompson y Hunston (2001); por último, se revisa el concepto de postura (stance).

\subsection{La teoría de la valoración de John Dewey}

Dewey (2008 [1977]: 139) plantea la siguiente tesis: "Ias valoraciones existen de hecho, y son susceptibles de observación empírica, de modo que las proposiciones acerca de ellas son verificables empíricamente", a pesar de las dificultades prácticas que reconoce que existen para la verificación. Lo anterior queda demostrado tras la elaboración de tres tipos o acepciones de la valoración: la expresión de valor como interjección, la valoración como agrado y desagrado, y las proposiciones de evaluación.

La expresión de valor como interjección es una tesis que Dewey (2008 [1977]) rechaza. Según esta tesis, dichas expresiones solo manifiestan sentimientos o pretenden suscitar sentimientos $y$, por tanto, no enuncian nada, equivalen simplemente a un tono de voz o un gesto. Este sería el caso en una frase como la siguiente:

(1) Hiciste mal en robar ese dinero.

En el ejemplo (1), hiciste mal equivale a la interjección, que se podría expresar con un tono de voz de desaprobación. Si bien Dewey (2008 [1977]) reconoce que existen fenómenos, como lágrimas o sonrisas de infantes, que no enuncian nada, ya que son simplemente signos de un estado orgánico, postula que llega un momento en el que estos signos se convierten en signos lingüísticos, porque se usan para inducir un comportamiento y, por tanto, no solo dicen algo, sino que, además, pretenden decir algo. Cuando la actitud corporal, como el llanto del infante, es intencionada, no se debería considerar como un sentimiento sino como comportamiento lingüístico. Por consiguiente, es superfluo hablar de sentimientos en este caso, como lo es para hablar de fenómenos que solo son abiertos a la introspección y no se pueden usar para explicar hechos. En cuanto a lo que comúnmente se llama "valor como interjección”, Dewey (2008 [1977]) afirma que consiste en un enunciado que contiene proposiciones verificables. Si alguien grita:

(2) ¡Fuego!

enuncia tres hechos observables y, por tanto, verificables empíricamente: a) que existe una situación que será perjudicial; b) que el enunciador no puede dominar esta situación; y c) que se prevé que la situación mejorará con el auxilio de otros. 
La valoración como agrado y desagrado también es un hecho verificable para Dewey (2008 [1977]). El autor considera el agrado en la acepción de ‘interesarse por', 'cuidar de', 'velar por' o 'atender', todos ellos verbos que corresponden a comportamientos observables. Distingue, entonces, el agrado del sentido ambiguo de 'disfrutar', que puede corresponder o bien a una situación de gratificación accidental o a una actividad de deleitarse en el esfuerzo por perpetuar la existencia de situaciones de gratificación. Sin embargo, esta segunda acepción de 'disfrutar' también se puede verificar empíricamente a través de la observación del comportamiento dedicado al esfuerzo. Y, como el esfuerzo es observable y, por ende, comprobable, también se puede usar para verificar la existencia de un deseo; no del deseo meramente personal (apetencia o fantasía), sino del deseo que surge en un contexto existencial debido a una carencia. Tanto la carencia como el esfuerzo por subsanarla son observables. Otro fenómeno relacionado con el agrado es el interés, el cual equivale a un conjunto de deseos interrelacionados. De nuevo, las acciones que se llevan a cabo para conseguir las condiciones que producirán las consecuencias deseadas son observables.

En cuanto a las proposiciones de evaluación con la acepción de 'estimación', estas se pueden ver realizadas en expresiones de precios, como en el ejemplo (3).

(3) El jugo cuesta mil pesos.

El ejemplo (3) enuncia una regla para la determinacón de un acto futuro y establece condiciones para alcanzar fines contemplados. También se refieren al futuro las reglas de valoración de los modos de comportamiento como sensatos/insensatos, eficaces/ineficaces, útiles/inútiles, etc., ya que pueden enunciar relaciones entre medios y fines verificables. Es decir, un comportamiento se puede valorar como ineficaz a través de la observación y modificarse para el futuro. Dicha valoración se puede aplicar tanto a medios como a fines.

El autor llega a la conclusión de que "Ios seres humanos hacen valoraciones continuamente" (Dewey, 2008 [1977]: 140), situando así el sujeto dentro del discurso con una presencia muy intensa, la cual, en la mayoría de los casos, se expresa de maneras verificables empíricamente. Además, asigna un papel importante al deseo de influir sobre el otro y coloca fuera del campo del estudio empírico los sentimientos solo susceptibles de introspección. Si bien los autores que serán revisados a continuación no rechazan en absoluto el uso del término "sentimientos", sin decirlo explícitamente, estudian los sentimientos que, según Dewey (2008 [1977]), se pueden observar empíricamente. Por último, la influencia sobre el otro obtiene un papel fundamental en todos los demás autores que se revisarán.

\subsection{La subjetividad en la escuela francesa del análisis del discurso}

En la escuela francesa del análisis del discurso se hace patente tanto el deseo de influir sobre el otro como la presencia del sujeto en el discurso a través del fuerte vínculo que posee con la sociedad y el lenguaje. El discurso, según Benveniste (1976), es lenguaje en acción y esta ac- 
ción se materializa necesariamente entre participantes, es decir, sujetos. Este autor rechaza la idea del lenguaje en cuanto herramienta como simplista en lo que respecta al sujeto del lenguaje. En el discurso siempre hay un yo y un tú, un yo que se dirige a un tú. Estas son las dos personas que se hacen cargo del discurso; las demás son no-personas. Para Benveniste (1976), el vínculo entre subjetividad (el posicionarse como sujeto) y lenguaje es tal que duda de si pudiera existir lenguaje sin subjetividad. La subjetividad es "la emergencia en el ser de una propiedad fundamental del lenguaje. Es 'ego' que dice 'ego'” (Benveniste, 1976: 260) y ahí es donde yace el fundamento de la subjetividad, la cual "se determina por el estatus lingüístico de la 'persona'” (Benveniste, 1976: 260). Visto desde la mirada de Althusser (2014), el discurso siempre produce un efecto de subjetividad. Ahora bien, como ya se mencionó, ese yo, para la escuela francesa, quiere influir sobre el tú, el otro. Si el otro presenta un obstáculo, o bien se elimina el obstáculo, o se tiene que convencer al otro (Charaudeau, 2005). Y para convencer, se activan varias estrategias que dejan la huella del sujeto en el discurso.

A un nivel de expresión lingüística, las marcas de subjetividad que señala Benveniste (1976, 2014) son: Ios pronombres personales, cuya importancia es primordial en todas las lenguas, ya que "no remiten ni a un concepto ni a un individuo" (Benveniste, 1976: 261), son, en definitiva, una clase de palabras con un estatus diferente de todas las demás, que remiten a la realidad del discurso; los otros deícticos; la temporalidad, ya que el tiempo lingüístico es autorreferencial, es siempre el tiempo en función del sujeto hablante; la interrogación; las órdenes; la aserción, tanto el tono asertivo como las expresiones de aserción, como el sí; los modos que expresan deseo, aprensión, posibilidad o indecisión; y los verbos como "creer", como "suponer", que implican una actitud hacia el enunciado, o como "jurar" y "prometer", que expresan un acto individual con impacto social, un acto de habla, como diría Austin (1981 [1955]). Nótese que ambos escritores destacan el hecho de que estos actos solo se realizan en primera persona. Maingueneau (2014), por otro lado, observa que para el yo y el tú la lengua no posee formas de sustitución, como las posee para las no-personas.

En cuanto a la subjetividad en el discurso, dentro de la escuela francesa cobra especial importancia el concepto de ethos de Maingueneau (2002), un concepto que remonta a la retórica de Aristóteles y que ha sido retomado y reelaborado por varios autores. Para Aristóteles, el ethos, el pathos y el logos constituyen los tres modos de persuadir. El pathos se refiere a las pasiones, tales como la ira y la calma, el amor y el odio, el temor, la vergüenza, la compasión y la envidia, que hacen que los hombres cambien sus juicios. El logos se refiere a un conjunto de conceptos, como la razón, el razonamiento y el discurso, mientras que el ethos, al carácter del orador, a la imagen que se crea de él en el discurso para poder influir en la audiencia (Amossy, 2001). Para poder persuadir a la audiencia, ella debe poder ver en el orador a alguien con el mismo ethos que ella (Maingueneau, 2002).

Maingueneau (2002) retoma el concepto de ethos de Aristóteles con sus tres características que se pueden situar en el marco del análisis del discurso: a) el ethos es un concepto discur- 
sivo y se construye por medio del discurso; b) está asociado a un proceso interactivo de influencia sobre el otro; y c) es un concepto sociodiscursivo. Según Maingueneau (2002), el ethos permite reflexionar sobre el proceso de la adhesión de los sujetos a cierto posicionamiento. Este autor ve la subjetividad, que se manifiesta por medio del discurso como una voz — mejor dicho un tono, que se aplica tanto a lo oral como a lo escrito-, asociada a un cuerpo históricamente especificado, situado. Además de ese tono, todos los textos tienen un garante y se inscriben en un mundo ético. El garante, que no necesariamente es una persona natural, es quien acredita lo dicho, y el mundo ético, el conjunto de los estereotipos culturales. ¿Por qué esa necesidad de ir más allá del sujeto a la noción del garante y todo un mundo ético? Sirva de ejemplo el caso de un enunciado en el que no está presente el enunciador. Hay textos jurídicos y científicos cuyo garante no es el autor físico del texto sino la ley o la ciencia; incluso el autor físico puede estar ausente, pero siempre habrá un garante y un mundo ético. Asimismo, a través del ethos, el destinatario se sitúa en la escena de enunciación. Para Maingueneau (2002), la escena de enunciación se analiza en tres escenas: la escena englobante, que proporciona al discurso su estatus pragmático, lo integra en un tipo como administrativo, filosófico, etc.; la escena genérica, que sitúa el discurso en un subgénero, como editorial o sermón; y la escenografía, la cual no viene impuesta por el género sino que se construye por el propio texto (un sermón puede, por ejemplo, ser profético o amistoso, según los ejemplos del autor). Como dice Maingueneau (2002: 17), "desde el momento en el que hay una enunciación, algo del orden del ethos se libera".

\subsection{La valoración en la LSF}

En esta sección se presenta el concepto de valoración en la LSF, desarrollado por Martin y White (2005). La valoración se inscribe en el sistema interpersonal en el nivel de la semántica discursiva. El sistema interpersonal es el relacionado con la representación de nuestra experiencia en la interacción social, donde el significado del lenguaje es un modo de actuar, que, a su vez, está relacionado con los participantes y los procesos. En este sistema, se concibe el lenguaje como una interacción entre el hablante y el oyente ${ }^{1}$, y el significado se activa por los rasgos del tenor, es decir, la identidad y la naturaleza de los participantes, concretamente sus estatus, papeles y relaciones (Halliday y Matthiessen, 2004). La valoración, situada en este nivel, coarticula el significado interpersonal con otros dos sistemas, es decir, otros dos recursos semánticos: la negociación y el involucramiento. Estos tres sistemas están relacionados con el poder y la solidaridad (Martin y White, 2005).

A su vez, la valoración consiste en tres regiones interrelacionadas: la actitud, la conexión y la graduación, cada una analizada en varias subregiones. La actitud se despliega en afecto,

1 Por "hablante” se entenderá tanto 'hablante' como 'escritor' en género genérico (masculino y femenino) y por “oyente” se entenderá tanto ‘oyente’ como ‘lector’ también en género genérico. 
juicio y aprecio; el compromiso, en monoglosia y heteroglosia; y la graduación, en fuerza y foco. Respecto a la conexión y la graduación, se trata de recursos lingüísticos con los que se toma una postura acerca de las posturas de valor en el texto y acerca de las personas a las que se dirigen. Para Martin y White (2005), tal como para la escuela francesa, todo enunciado contiene una postura o actitud. La conexión se refiere al grado en el que se reconocen los hablantes anteriores y cómo se establece una conexión o relación con ellos (de ahí los conceptos de monoglosia y heteroglosia de Bakhtin [2014]). La graduación se refiere al grado de prototipicidad (foco) y el de intensidad y cantidad (fuerza) de todos los significados (subregiones) de la actitud (Martin y White, 2005). A continuación, se profundiza en la región de la actitud con sus tres subregiones.

La primera región de la actitud que se revisará es el afecto, la región de los sentimientos y las reacciones emocionales a comportamientos, textos, procesos, fenómenos. El afecto está en el núcleo de la actitud por su naturaleza innata y se clasifica en términos de seis factores, donde aparece un tipo de participante consciente que experimenta una emoción, el Emocionador, así como un fenómeno responsable por la emoción, el Gatillador. El primer factor es la interpretación popular como algo positivo o negativo, como la interpretación popular de "contento" y “triste”. El segundo es la emoción que involucra algún tipo de manifestación paralingüística o extralingüística corpórea, o la emoción experimentada internamente como un estado emotivo o un proceso mental. Una manifestación extralingüística corpórea sería el proceso conductual en el ejemplo (4).

(4) le sonrió

Un estado emotivo, que correspondería a un proceso mental, sería el ejemplo (5).

(5) él le cayó bien

Y un proceso mental sería el proceso relacional del ejemplo (6).

(6) se sentía feliz con él

El tercer factor consiste en los sentimientos dirigidos hacia un Gatillador o los que reaccionan ante un Gatillador, o sentimientos que corresponden a un estado de ánimo por cuya causa el Emocionador puede no estar seguro. En el ejemplo (7) hay dos participantes, el Sensor, que aquí es el Emocionador, y el Fenómeno, es decir, el Gatillador.

(7) ella lo quiere

El Emocionador es “ella” y el Gatillador es él (“Io”). En cambio, en el ejemplo (8) se expresa un estado relacional por cuya causa el Emocionador podría responder que la desconoce.

(8) estoy contenta 
El cuarto factor de clasificación del afecto es la graduación de sentimientos en una escala de intensidad, en la que el nivel bajo se expresaría en el ejemplo (9), el mediano en el (10) y el alto en el (11).

(9) no me gusta la violencia

(10) odio la violencia

(11) aborrezco la violencia

El quinto factor consiste en si los sentimientos involucran una intención en cuanto a un estímulo realis (“me gusta”) o una reacción a un estímulo irrealis ("me gustaría”). El último factor es una clasificación de las emociones en tres categorías: felicidad/infelicidad, seguridad/inseguridad, satisfacción/insatisfacción. Cabe destacar la importancia de la sociedad, ya que en la elaboración de la valoración por Martin y White (2005) están continuamente presentes los orígenes sociosemióticos de la LSF. En este caso, se subraya que las emociones de seguridad y satisfacción, en las sociedades con un tratamiento estereotipado del género, se suelen asociar con la protección maternal y con el comportamiento del padre o mentor, respectivamente. La protección maternal en la casa (mothering) incluye sentimientos de paz y ansiedad en relación con el entorno, y el comportamiento del padre (fathering) está enfocado en el aprendizaje y el logro, por lo que está relacionado con el cumplimiento de objetivos y, por tanto, con los sentimientos de logro y frustración que ello conlleva.

La segunda región de la actitud, el juicio, corresponde a los recursos para evaluar comportamientos. Los juicios son actitudes ante comportamientos que se admiran, critican, condenan, etc. Se pueden clasificar bajo la categoría de la estima o la sanción social. La estima social incluye la normalidad, la capacidad y la tenacidad de las personas (cuán normal, capaz o firme es alguien) y, en la cultura oral, se monitorea a través de las conversaciones denominadas tradicionalmente "cotilleo" o el humor. La sanción social se clasifica bajo el rótulo de la veracidad o la decencia ética (cuán sincero y cuán ético es alguien) y se codifica más por escrito en reglas y leyes. Se suele monitorear por instituciones como la Iglesia y el Estado (los aparatos ideológicos del Estado, según Althusser [1970]), las cuales prevén penalizaciones para los que no cumplen con el código. En definitiva, el juicio corresponde a cómo deberíamos comportarnos y tiene su correlato filosófico en la ética.

La tercera región de la actitud, el aprecio, consiste en los recursos para interpretar el valor de las cosas. Se trata de evaluaciones de fenómenos semióticos y naturales en cuanto a los modos en los que se aprecian o no en un campo dado. El valor se puede formalizar en precios o premios, pero también puede ser el grado de innovación, adecuación, autenticidad, etc. El aprecio tiene su correlato filosófico en la estética y su equivalente en la teoría de Dewey (2008 [1977]) en la apreciación.

Respecto a las tres regiones de la actitud cabe destacar, por un lado, que las tres implican una postura del hablante y, por otro, que si bien el afecto se considera innato, el juicio y el 
aprecio se consideran como sentimientos institucionalizados y se aprenden en la casa desde las primeras etapas del desarrollo lingüístico (Martin y White, 2005). Esta última afirmación es otra manifestación de los orígenes sociosemióticos de la LSF, ya que desde un principio se postulaba la inseparabilidad del hombre social, su conducta y la lengua. Para Halliday (1982 [1978]), el organismo humano se transforma en ser social mediante procesos lingüísticos, ya que aprende a la vez el comportamiento y la lengua y el uno a través del otro.

\subsection{La evaluación según Thompson y Hunston}

Otro concepto que pretende abarcar los fenómenos contemplados por Martin y White (2005) es el de evaluación, tal como fue desarrollada por Thompson y Hunston (2001). Estos autores coinciden con Martin y White (2005) en la aproximación funcional de la descripción de la lengua en uso y, por eso, optan por una perspectiva centrada en el usuario, no en los ítems léxicos (Thompson y Hunston, 2001). Esto se ve reflejado ante todo en la selección del término "evaluación" entre la amplia gama terminológica usada para la expresión de la subjetividad. Los autores prefieren el uso del término "evaluación”, por un lado, porque expresa la perspectiva del usuario (es el usuario quien evalúa) y, por otro, porque es un término flexible sintáctica y morfológicamente. Declaran que la evaluación cubre la expresión de actitud, postura, punto de vista, sentimientos y opinión sobre proposiciones y entidades. En este sentido, se ve cubierto el plano de subjetividad cubierto por la valoración de Martin y White (2005).

Thompson y Hunston (2001) proceden a dos distinciones acerca de la opinión. En primer lugar, distinguen entre dos tipos de opinión: a) juicio del tipo bueno-malo; y b) opiniones acerca de la probabilidad de eventos, lo que tradicionalmente se ha denominado "modalidad" y que presenta cierto solapamiento con el concepto de evidencialidad. En segundo lugar, distinguen entre opiniones expresadas sobre proposiciones y sobre entidades. Las primeras suelen realizarse en cláusulas, y las segundas, en grupos nominales (nótese aquí la terminología sistémico-funcionalista). Las opiniones sobre proposiciones suelen ser más gramaticalizadas y más integradas en la estructura de la cláusula, lo que queda ilustrado en los ejemplos (12) y (13), donde el primero expresa una opinión acerca de una proposición y el segundo, acerca de una entidad.

(12) ¡Qué bueno que hayas venido!

(13) Miguel es un genio.

Esta distinción les parece relevante, porque entre los dos tipos, a pesar de que existe un terreno común, suele haber ciertas regularidades de diferenciación, como, por ejemplo, que las opiniones de probabilidad por lo general se restringen a las proposiciones, no se aplican a entidades. Sin embargo, los dos tipos pueden coocurrir.

Según Thompson y Hunston (2001), la importancia de la evaluación para la lingüística se debe a las tres funciones que cumple: a) expresar opiniones y, por tanto, el sistema valórico del 
hablante así como de su comunidad; b) entablar y mantener relaciones con los lectores; y c) organizar el discurso. Estas tres funciones se detallan a continuación.

A primera vista, la expresión de opinión se refiere a los pensamientos y sentimientos del hablante. No obstante, los pensamientos y sentimientos del hablante no existen en un vacío; el hablante está sumergido en una sociedad, en una cultura, incluso en una subcultura, cuyos valores también expresa, tras haberse apropiado de ellos; y al expresarlos, los valida, los refuerza, alimentando así el sistema valórico de su comunidad. Según Thompson y Hunston (2001), cada acto de evaluación está dirigido hacia el fortalecimiento de ese sistema valórico, el cual, a su vez, es un componente de la ideología subyacente de cualquier texto. Para ilustrar esa idea, se propone el ejemplo (14) de Thompson y Hunston (2001).

(14) My husband runs his own business and is a workaholic. Last year he kept putting off our plans for a break and I got so fed up I went away with four friends. We had a great time, even though I missed him. I want a holiday for just us this year but he keeps saying I'd probably have a better time if I went with them. How can I get him to see I'd rather go with him?

En el ejemplo están marcados en cursiva los elementos que los autores consideran que expresan opinión. A primera vista, se detectan los sentimientos de la hablante, como, por ejemplo, que echó de menos a su esposo. Sin embargo, a un nivel más profundo, se pueden leer los supuestos ideológicos que subyacen en las opiniones de la hablante, tales como que es bueno tener vacaciones, no es bueno trabajar sin interrupción, las parejas deberían irse de vacaciones juntas, etc. Estos supuestos guardan una similitud significante con el concepto de juicio de Martin y White (2005), que expresa el cómo deberíamos comportarnos y que se puede ver implementado en reglas y leyes. En el ejemplo (14) se observa una relación con la noción de normalidad de Martin y White (2005). Ahora bien, aun habiendo detectado este segundo nivel de opinión, todavía hay uno más profundo: estos supuestos reflejan y refuerzan una ideología basada en el sistema económico y familiar. Según el sistema económico subyacente, las personas trabajan por necesidad, el trabajo es una parte separada de las otras partes de la vida, uno puede disfrutar de su trabajo pero no más que sus vacaciones, etc. Y, como dirían Althusser (1970) y Martin y White (2005), las personas empiezan a conocer este sistema desde su infancia a través de las instituciones de la familia y la escuela. Se observa, por tanto, en el pensamiento de Thompson y Hunston (2001) la idea tan recurrente en la escuela francesa y en la LSF del yo como miembro o parte de la sociedad.

En cuanto a la segunda función, que es entablar y mantener relaciones con los lectores, esta se ve reflejada en la manipulación o la persuasión (concepto ya presentado en su elaboración por Maingueneau [2002]), en el uso del hedging (cobertura), es decir, de expresiones que atenúan la naturaleza categórica de un enunciado, así como en la cortesía. En este caso, el papel de la evaluación es ajustar el valor de verdad o de certeza de un enunciado (Thompson y Hunston, 
2001). Por último, la tercera función, la organización del discurso, se plantea en términos de la relación entre autor-lector pero en el propio texto. Como observan Thompson y Hunston (2001), es como si el autor dijera "aquí empieza nuestro texto" o "aquí termina nuestra interacción".

La evaluación se expresa a nivel léxico y a nivel gramatical. Según Thompson y Hunston (2001), existen ítems léxicos cuya principal función es la evaluación, como los adjetivos "terrible" y "fantástico", o los sustantivos "éxito", "tragedia” y “triunfo", pero la expresión de la evaluación también puede depender del contexto. A nivel gramatical, la evaluación se puede expresar a través de imperativos, negativos, modales, preguntas, entre otros. Sin embargo, hay consenso en el área sobre el hecho de que la evaluación es algo que impregna el texto, que se halla en su totalidad y no en una parte concreta, aunque sí existe una tendencia a expresarse en ciertas partes específicas, que suelen ser los límites de las partes textuales (Thompson y Hunston, 2001).

\subsection{La postura}

El último concepto que se revisa en esta sección es el de la postura. Se trata de los mecanismos lingüísticos con los que se transmiten actitudes, sentimientos, opiniones y evaluaciones (Conrad y Biber, 2001; Gray y Biber, 2012), por lo que queda explícito el solapamiento con el término "evaluación" seleccionado por Thompson y Hunston (2001). Efectivamente, Gray y Biber (2012) afirman que la postura guarda relación con los conceptos de afecto, evidencialidad, hedging y evaluación. Tanto la valoración de Martin y White (2005) como la evaluación de Thompson y Hunston (2001) y la postura pretenden abarcar los mismos fenómenos. Las diferencias yacen sobre todo en las clasificaciones y los focos de énfasis.

Concretamente, Conrad y Biber (2001) proceden a la clasificación de la postura en tres tipos: epistémica, actitudinal y de estilo. La postura epistémica es la realizada a través de comentarios sobre la certeza, la confiabilidad o las limitaciones de una proposición, incluyendo los comentarios sobre la fuente de información. La actitudinal es la que transmite las actitudes, los sentimientos o los juicios de valor del hablante. La postura de estilo es la que describe el modo en el que se presenta la información. Estos tres tipos de postura tienen su correlato en las tres funciones de la evaluación de Thompson y Hunston (2001). La postura epistémica guarda similitud con la segunda función de la evaluación (entablar y mantener relaciones con los lectores), una parte de la cual corresponde a ajustar el valor de verdad o de certeza de un enunciado. La postura actitudinal encuentra su reflejo en la primera función de la evaluación, la expresión de opinión, que se refiere a los pensamientos y sentimientos del hablante. Y la postura de estilo guarda correspondencia a la tercera función, que es la organización del discurso.

\section{Los productos del PA: calidad y autoría}

En esta sección se revisa la bibliografía relacionada con los productos del PA. En una primera instancia, se aproxima su calidad. Se detallan sus características, acompañadas por ejemplos, 
y se revisa la literatura acerca de su calidad y cómo son percibidos por los humanos. Después, se procede a revisar el tema de la autoría de dichos productos.

\subsection{Características y calidad de los productos del PA y sobre cómo son perci- bidos por los humanos}

Los productos del PA se han caracterizado como mecánicos y repetitivos en cuanto a sus patrones lingüísticos (Fanta, 2017). Su calidad lingüística se considera inferior a la de la escritura humana y limitada, ya que carecen de matices típicos del lenguaje humano, como el humor, el sarcasmo y las metáforas (Graefe, 2016). Asimismo, se describen como técnicos y aburridos (Graefe, 2016), aunque se espera que su calidad va a mejorar con los avances tecnológicos (Graefe, 2016; Fanta, 2017). A continuación, se proporcionan dos ejemplos de productos de PA en inglés.

(15) Apple's holiday earnings for 2014 were record shattering. The company earned an \$18 billion in revenue. That profit was more than any company had ever earned in history. [Fragmento de artículo de Business Insider]

(16) On a per-share basis, the Bentonville, Arkansas-based company said it had proft of \$1. The results surpassed Wall Street expectations. The average estimate of 14 analysts surveyed by Zacks Investment Research was for earnings of 96 cents per share. [Fragmento de atículo proporcionado por Fanta (2017)]

En cuanto a la percepción de estos productos de parte de humanos, los autores coinciden en que las encuestas Ilevadas a cabo hasta el momento demuestran que no es fácil distinguir los artículos generados por máquinas y los generados por humanos (Fanta, 2017; Caswell y Dörr, 2018). A pesar de eso, sí hay diferencias en la evaluación de ambos. Los artículos escritos por humanos se califican como más agradables de leer (Graefe y otros, 2015; Graefe, 2016; Fanta, 2017), pero los artículos generados por máquinas se evalúan como más fiables (Graefe y otros, 2015; Graefe, 2016) y como artículos atribuibles a alguien más experto en materia periodística (Graefe y otros, 2015).

Un tema relacionado con la calidad de estos artículos es el de su edición por humanos antes de la publicación. Según Fanta (2017), una gran parte del contenido automatizado se publica sin edición humana. A modo de ilustración, se puede mencionar que Reuters publica 950 alertas y 400 artículos al día sin ninguna intervención humana, y no es la única agencia que opta por esa práctica (Fanta, 2017).

\subsection{Autoría en el PA}

Ahora bien, el fenómeno del PA no ha dejado a la comunidad periodística indiferente, ya que conlleva ramificaciones importantes a nivel práctico, sociopolítico, psicológico, legal y profesional, entre otras razones por el tema de la autoría de dichos productos (Montal, 2015), tema 
que también tiene su incidencia en la ética profesional y que abre el debate de la transparencia (Fanta, 2017). La ambigüedad sobre la autoría de los artículos del PA, aparte del problema filosófico y lingüístico que puede plantear, tiene un impacto en el asunto de los derechos de autor. Según la encuesta realizada por Fanta (2017), no siempre se menciona el uso de un robot en la redacción de los artículos: Reuters, AP y NTB suelen mencionarlo, pero hay agencias que producen artículos parcialmente constituidos por plantillas y no mencionan al robot como coautor.

El problema de si atribuir autoría a una máquina no es nuevo. Ya en 1964 se presentó una solicitud al Registro de Derechos de Autor de los EE. UU. por una composición musical creada por un computador, generando junto con otras solicitudes similares un rompecabezas para la institución. En ese momento, se planteó el criterio de distinción entre el uso del computador como asistente y la atribución de los elementos tradicionales de autoría a la máquina a nivel de concepción y ejecución. Desde entonces, varias instancias de regularización de los productos de computadores y de legislación sobre ellas han tenido lugar en varios países, como los EE. UU., el Reino Unido e India, dejando, sin embargo, abiertos interrogantes acerca de la coautoría, de si debería reconocerse como autor el programador, el algoritmo o el proveedor de los datos, así como de una posible ausencia de autoría, entre otros (Montal, 2015).

En la investigación realizada por Montal (2015), en cuanto al tema de quién consideran los expertos autor de las noticias automatizadas, el factor humano prevaleció. Todos los entrevistados coincidieron en que es un humano quien debería considerarse como autor, sea el organismo o los involucrados en la programación y la compilación de los datos. Nadie tuvo la menor predisposición de aceptar al computador o al algoritmo como autor. Sin embargo, Montal (2015) subraya que en el campo de la inteligencia artificial hay voces que proclaman el carácter de entidad performativa de los algoritmos, abogando que constituyen sustitutos de la inteligencia humana en varias tareas cognitivas y que poseen capacidad autónoma de toma de decisiones así como la capacidad de aprendizaje. Sin embargo, el punto álgido de la creatividad (imaginación y consciencia) queda sin consenso en el área (Montal, 2015).

La identificación del autor con una persona física transmitida por los entrevistados de Montal (2015) coincide con la solución legal en caso de errores, ya que un algoritmo no tiene responsabilidad legal (Graefe, 2016). Sin embargo, la transparencia en cuanto a la autoría de los artículos será fundamental en estos casos (Graefe, 2016). Este tema práctico y legal, junto con los demás temas éticos relacionados con la autoría, hacen que sean urgentes las respuestas a las preguntas abiertas que se presentaron en esta sección.

\section{Discusión}

La revisión realizada en la sección 3 sobre el concepto de subjetividad en el discurso desde el análisis del discurso permite la descomposición de este concepto en varios elementos no de menor importancia. Se desprende que no se trata simplemente de un yo, de la existencia de un sujeto en 
un vacío, porque el sujeto, para que sea sujeto, necesita de los demás componentes de la subjetividad. A continuación, se retoman los componentes de la subjetividad vistos desde una perspectiva global de los diferentes enfoques estudiados con comentarios acerca de su presencia en los productos del PA, según la bibliografía relevante. De esta manera, posteriormente, se llega a una conclusión acerca de si estos productos se pueden considerar instancias de discurso.

En primer lugar, aparece de manera inevitable el sujeto, el yo, un ethos construido en el discurso. Este primer y fundamental componente no está presente en los artículos del PA. En segundo lugar, aparece el componente tú, el otro, sobre el cual el yo quiere ejercer cierta influencia (persuasión, etc.). El tú sí está presente en el PA: el lector del artículo. También está presente la intención de influencia de parte de la empresa o agencia de noticias. Otro componente es el del tiempo, el tiempo autorreferencial del que habla Benveniste (1976). Aunque el tiempo se determina en función del hablante y el hablante es ausente en el PA, no se pueden considerar los artículos generados por computador fuera del tiempo; el tiempo de referencia en este caso es el tiempo de publicación.

No obstante, aparte del sujeto o más allá del sujeto, hay un trasfondo que no se puede no tener en cuenta, porque, como ya se ha explicado, el sujeto no está en un vacío sino situado en una sociedad/comunidad/cultura/subcultura. Aquí se puede descomponer el sujeto en dos planos: el personal/individual y el trasfondo ideológico. El personal consiste en las opiniones personales y el sistema valórico del sujeto. El trasfondo ideológico corresponde a lo que Maingueneau (2002) denomina mundo ético, lo que Thompson y Hunston (2001) Ilaman supuestos ideológicos y sistema valórico de la comunidad y lo que Martin y White (2005) ven como interpretación popular, estereotipos de género y sentimientos institucionalizados. El primer plano no está presente en el PA al carecer este de sujeto. Sin embargo, el segundo es inevitable. Nada se puede construir o generar que no esté impregnado de la ideología subyacente de la sociedad. Incluso los artículos del PA son generados a partir de muestras de lenguaje generadas por humanos con un trasfondo ideológico. Además, los temas son seleccionados de manera directa o indirecta por la agencia, que también se basa en una ideología. La agencia o empresa de noticias es el garante de Maingueneau (2002), quien tiene prevista la posibilidad de un texto sin sujeto presente pero siempre con un garante. Por tanto, en el caso del PA, falta uno de los planos, el personal/individual, pero la base (la ideología) está presente.

Otros componentes de la subjetividad son la expresión de certeza o evidencia y la organización del discurso. Para determinar la presencia de estos componentes en los productos del PA, sería necesario llevar a cabo un estudio empírico, pero se puede argumentar que estos dos componentes se materializan a través de expresiones lingüísticas que la máquina puede generar a través de las plantillas y que, si todavía no se generan a nivel satisfactorio, sí forman parte de los objetivos de los programadores y son de los elementos textuales mejorables con los avances tecnológicos.

Por último, cabe revisar el concepto de escena de enunciación de Maingueneau (2002) para determinar la presencia o ausencia de cada una de sus subescenas en el PA. La escena en- 
globante se ve realizada, ya que los productos del PA pertenecen a un tipo concreto, el periodístico. La escena genérica, que sitúa el discurso en un subgénero, también se ve realizada, porque los productos del PA pertenecen a un subgénero concreto, como, por ejemplo, artículo o alerta. La escenografía, que es la que se construye por el propio texto, necesitaría de un sujeto para realizarse. Lo hasta aquí expuesto sobre los componentes de la subjetividad y su presencia en el PA se resume en la tabla 1.

\section{TABLA 1}

Componentes de subjetividad y su presencia en el PA

\begin{tabular}{lc}
\multicolumn{1}{l}{ COMPONENTE DE SUBJETIVIDAD } & PRESENCIA EN PA \\
\hline yo & no \\
\hline tú & sí \\
\hline tiempo & sí \\
\hline garante & sí \\
\hline trasfondo ideológico & sí \\
\hline influencia & sí \\
\hline organización del discurso & - \\
\hline certeza/evidencia & - \\
\hline escena englobante & sí \\
\hline escena genérica & sí \\
\hline escenografía & no
\end{tabular}

Se desprende de los resultados que los productos del PA cumplen con la mayoría de los requisitos, es decir, presentan la mayoría de los componentes fundamentales de la subjetividad, para ser llamados textos, instancias de discurso. Se puede considerar, por tanto, que, a pesar de la ausencia de algunos de los componentes, pueden pertenecer a la categoría del discurso sin ser ejemplares prototípicos (Lakoff, 1987). Además, si no hubiera ningún rasgo de subjetividad en estos artículos, no los percibiríamos como lenguaje (Benveniste, 1976) y, sin embargo, sí se consideran como lenguaje que ni siquiera se puede distinguir del humano (Fanta, 2017; Caswell y Dörr, 2018). Se concluye, por ende, que los productos del PA constituyen discurso, aunque no un ejemplar prototípico de él.

\section{Conclusiones}

La revisión realizada en el presente artículo permitió la descomposición del concepto de subjetividad para considerar cada uno de los componentes como requisito para que una instancia de lenguaje sea considerada como discurso. De los componentes aquí identificados, se pudo 
determinar su presencia en los productos del PA para la gran mayoría, lo que permitió la caracterización de dichos productos como instancias de discurso, aunque no como ejemplares prototípicos de él. Sin embargo, hay dos componentes, la organización del discurso y la expresión de certeza o evidencialidad, cuya presencia en el PA no se pudo determinar a nivel teórico. Para ello, se necesitarán estudios empíricos de análisis de artículos generados por computador. Un estudio empírico de comparación entre artículos escritos por humanos y artículos generados por computadores permitiría, además, profundizar más en la naturaleza de los segundos y los resultados se podrían relacionar con los que ya existen sobre cómo los perciben los humanos. Por último, cabe subrayar que los resultados de investigaciones sobre los productos del PA se pueden contrastar y complementar con resultados sobre productos de otro tipo de sistemas de generación de lenguaje natural; esto puede incluso contribuir a su caracterización.

\section{Bibliografía citada}

Althusser, Louis, 1970: "Idéologie et appareils idéologiques d'état”, La Pensée, París: Les éditions sociales, 67-125

Althusser, Louis, 2014: "The subjectivity effect of discourse" en Johannes Angermuller, Dominique Maingueneau y Ruth Wodak (eds.): The Discourse Studies Readers. Main currents in theory and analysis, Amsterdam: Benjamins, 83-88.

Amossy, Ruth, 2001: "Ethos at the Crossroad of Disciplines: Rhetoric, Pragmatics, Sociology", Poetics Today 22 (1), 1-23.

Angermuller, Johannes, Dominique Maingueneau y Ruth Wodak, 2014: "An Introduction" en Johannes Angermuller, Dominique Maingueneau y Ruth Wodak (eds.): The Discourse Studies Readers. Main currents in theory and analysis, Amsterdam: Benjamins, 17-20.

Aristóteles, Retórica, Libro II.

Austin, John, 1981 [1955]: Cómo hacer cosas con palabras, España: Paidós.

Bakhtin, Mikhail, 2014: "Polyphonic discourse in the novel" en Johannes Angermuller, Dominique Maingueneau y Ruth Wodak (eds.): The Discourse Studies Readers. Main currents in theory and analysis, Amsterdam: Benjamins, 27-35.

Benveniste, Émile, 1976: Problèmes de linguistique générale. Tome 1, París: Gallimard.

Benveniste, Émile, 2014: "The formal apparatus of enunciation” en Johannes Angermuller, Dominique Maingueneau y Ruth Wodak (eds.): The Discourse Studies Readers. Main currents in theory and analysis, Amsterdam: Benjamins, 140-145. 
Caswell, David, y Konstantin DörR, 2018: "Automated Journalism 2.0: Event-driven narratives. From simple descriptions to real stories”, Zurich Open Repository and Archive, Universidad de Zurich. DOl: https://doi.org/10.1080/17512786.2017.1320773 [https://www.zora.uzh. ch/137060/1/Automated_Journalism_2.0_EventDriven_Narratives.pdf, fecha de consulta: 25 de octubre de 2018].

Charaudeau, Patrick, 2005: "Lenguaje, acción, poder. De la identidad social a la identidad discursiva del sujeto" [http://www.patrick-charaudeau.com/Lenguaje-accion-poder-De-la.html, fecha de consulta: 22 de septiembre de 2018].

Conrad, Susan, y Douglas Biber, 2001: "Adverbial Marking of Stance in Speech and Writing" en Susan Hunston y Geoff Thompson (eds.): Evaluation in Text. Authorial Stance and the Construction of Discourse, Nueva York: Oxford, 56-73.

DEWEy, John, 2008 [1977]: Teoría de la valoración, Madrid: Siruela.

FAnTA, Alexander, 2017: "Putting Europe's Robots on the Map: Automated journalism in news agencies", Reuters Institute for the Study of Journalism, Universidad de Oxford [https://reutersinstitute.politics.ox.ac.uk/sites/default/files/2017-09/Fanta\%2C\%20Putting\%20Europe's\%20 Robots\%200n\%20the\%20Map.pdf, fecha de consulta: 25 de octubre de 2018].

Graefe, Andreas, 2016: "Guide to Automated Journalism”, Tow Center for Digital Journalism [http://towcenter.org/research/guide-to-automated-journalism/, fecha de consulta: 25 de octubre de 2018].

Graefe, Andreas, Mario Haim, Bastian Haarmann y Hans-Bernd Brosius, 2015: "Perception of Automated Computer-Generated News: Credibility, Expertise, and Readability”, comunicación presentada en IIth Dubrovnik Media Conference Days: Artificial Intelligence, Robots, and Media.

Gray, Bethany, y Douglas Biber, 2012: "Current Conceptions of Stance" en Ken Hyland y Carmen Sancho GuIndA (eds.): Stance and Voice in Written Academic Genres, Londres: Palgrave MacmiIlan, 15-33.

Halliday, Michael, 1982 [1978]: El lenguaje como semiótica social: Ia interpretación social del lenguaje y del significado, México: Fondo de Cultura Económica.

Halliday, Michael, y Christian Matthiessen, 2004: An introduction to functional grammar, Nueva York: Hodder Arnold.

LakofF, George, 1987: Women, Fire, and Dangerous Things. What Categories Reveal about the Mind, Chicago y Londres: The University of Chicago Press. 
Leppännen, Leo, Myriam Munezero, Mark Granroth-Wilding y Hannu Toivonen, 2017: "Data-Driven News Generation for Automated Journalism", Proceedings of the 1oth International Conference on Natural Language Generation, DOI: 10.18653/v1/W17-3528 [https://www.cs.helsinki. fi/u/htoivone/pubs/leppanenetal_inlg_2017.pdf, fecha de consulta: 25 de octubre de 2018].

Maingueneau, Dominique, 2002: “L'ethos, de la rhétorique à l'analyse du discours. Version raccourcie et légèrement modifiée de 'Problèmes d'ethos'”, Pratiques No. 113-114 [http:// dominique.maingueneau.pagesperso-orange.fr/pdf/Ethos.pdf, fecha de consulta: 25 de octubre de 2018].

Maingueneau, Dominique, 2014: "The scene of enunciation" en Johannes Angermuller, Dominique MaIngueneAu y Ruth Wodak (eds.): The Discourse Studies Readers. Main currents in theory and analysis, Amsterdam: Benjamins, 146-154.

Martin, James, y Peter White, 2005: The Language of Evaluation. Appraisal in English, Nueva York: Palgrave Macmillan.

MontAL, Tal, 2015: I, robot. You, Journalist. Who is the author? Authorship, bylines and full disclosure in automated journalism. Tesis de Maestría, Universidad Ben-Gurion de Negev.

Reiter, Ehud, y Robert Dale, 2000: Building Natural Language Generation Systems, Reino Unido: Cambridge University Press.

Thompson, Geoff, y Susan Hunston, 2001: “Evaluation: An Introduction” en Susan Hunston y Geoff Thompson (eds.): Evaluation in Text. Authorial Stance and the Construction of Discourse, Nueva York: Oxford University Press, 1-27. 